\title{
Probability 1 computation with chemical reaction networks*
}

\author{
Rachel Cummings ${ }^{\dagger} \quad$ David Doty ${ }^{\ddagger} \quad$ David Soloveichik ${ }^{\S}$
}

\begin{abstract}
The computational power of stochastic chemical reaction networks (CRNs) varies significantly with the output convention and whether or not error is permitted. Focusing on probability 1 computation, we demonstrate a striking difference between stable computation that converges to a state where the output cannot change, and the notion of limit-stable computation where the output eventually stops changing with probability 1 . While stable computation is known to be restricted to semilinear predicates (essentially piecewise linear), we show that limit-stable computation encompasses the set of predicates in $\Delta_{2}^{0}$ in the arithmetical hierarchy (a superset of Turing-computable). In finite time, our construction achieves an error-correction scheme for Turing universal computation. This work refines our understanding of the tradeoffs between error and computational power in CRNs.
\end{abstract}

${ }^{*}$ The first author was supported by NSF grants CCF-1049899 and CCF-1217770, the second author was supported by NSF grants CCF-1219274 and CCF-1162589 and the Molecular Programming Project under NSF grant 1317694, and the third author was supported by NIGMS Systems Biology Center grant P50 GM081879.

${ }^{\dagger}$ Northwestern University, Evanston, IL, USA, rachelc@u.northwestern. edu

${ }^{\ddagger}$ California Institute of Technology, Pasadena, CA, USA, ddoty@caltech. edu

${ }^{\S}$ University of California, San Francisco, San Francisco, CA, USA, david.soloveichik@ucsf.edu 


\section{Introduction}

Recent advances in the engineering of complex artificial molecular systems have stimulated new interest in models of chemical computation. How can chemical reactions process information, make decisions, and solve problems? A natural model for describing abstract chemical systems in a well-mixed solution is that of (finite) chemical reaction networks (CRNs), i.e., finite sets of chemical reactions such as $A+B \rightarrow A+C$. Subject to discrete semantics (integer number of molecules) the model corresponds to a continuous time, discrete state, Markov process. A state of the system is a vector of non-negative integers specifying the molecular counts of the species (e.g., $A, B, C)$, a reaction can occur only when its reactants are present, and transitions between states correspond to reactions (i.e., when the above reaction occurs the count of $B$ is decreased by 1 and the count of $C$ increased by 1). CRNs are used extensively to describe natural biochemical systems in the cellular context. Other natural sciences use the model as well: for example in ecology an equivalent model is widely employed to describe population dynamics [18]. Recently, CRNs began serving as a programming language for engineering artificial chemical systems. In particular, DNA strand displacement systems have the flexibility to realize arbitrarily complex interaction rules $[5,7,17]$, demonstrating that arbitrary CRNs have a chemical implementation. Outside of chemistry, engineering of sensor networks and robot swarms often uses CRN-like specification rules to prescribe behavior [3].

The exploration of the computational power of CRNs rests on a strong theoretical foundation, with extensive connections to other areas of computing. Similar models have arisen repeatedly in theoretical computer science: Petri nets [12], vector addition systems [11], population protocols [3], etc. They share the fundamental feature of severely limited "agents" (molecules), with complex computation arising only through repeated interactions between multiple agents. In population protocols it is usually assumed that the population size is constant, while in CRNs molecules could be created or destroyed ${ }^{1}$ - and thus different questions are sometimes natural in the two settings.

Informally speaking, we can identify two general kinds of computation in CRNs. In non-uniform computation, a single CRN computes a function over a finite domain. This is analogous to Boolean circuits in the sense that any given circuit computes only on inputs of a particular size (number of bits), and to compute on larger inputs a different circuit is needed. Conversely, in uniform computation, a single CRN computes on all possible input vectors. This is analogous to Turing machines that are expected to handle inputs of arbitrary size placed on their (unbounded) input tape. In this work we focus entirely on uniform computation. ${ }^{2}$

We focus on the question of the distinguishability of initial states by a CRN. We view CRNs as computing a Boolean valued predicate on input vectors in $\mathbb{N}^{k}$ that are the counts of certain input species $X_{1}, \ldots, X_{k}$. We believe that a similar characterization holds for more general function computation where the output is represented in counts of output species (e.g. $f: \mathbb{N}^{k} \rightarrow \mathbb{N}^{l}$ ), but that remains to be shown in future work.

Previous research on uniform computation has emphasized the difference in computational power between paradigms intended to capture the intuitive notions of error-free and error-prone computation [8]. In contrast to many other models of computing, a large difference was identified between the two settings for CRNs. We now review the previously studied error-free (probability 1 ) and error-prone (probability $<1$ ) settings. In this paper we develop an error correction scheme that reduces the error of the output with time and achieves probability 1 computation in the limit.

\footnotetext{
${ }^{1}$ Having an external (implicit) supply of fuel molecules avoids violating the conservation of mass.

${ }^{2}$ However, it is important to keep in mind that settings with extremely weak uniform computational power may nonetheless achieve complex computation from the non-uniform perspective. For example, probability 1 committing computation (see below), while restricted to constant predicates in our setting, can simulate arbitrary Boolean circuits with a proper encoding of input and output (e.g. using so called "dual-rail" encoding with two species per input bit).
} 


\begin{tabular}{l|c|c|c|} 
& committing & stable & limit-stable \\
\hline prob correct $=1$ & $($ constant $)$ & semilinear $[1]$ & $\Delta_{2}^{0}[$ this work] \\
\hline prob correct $<1$ & computable $[16]$ & $($ computable $)$ & $\left(\Delta_{2}^{0}\right)$ \\
\hline
\end{tabular}

Table 1: Categorizing the computational power of CRNs by output convention and allowed error probability. In all cases we consider the class of total predicates $\phi: \mathbb{N}^{k} \rightarrow\{$ no, yes $\}$ ("total" means $\phi$ must be defined on all input values). The input consists of the initial molecular counts of input species $X_{1}, \ldots, X_{k}$. Committing: In this output convention, producing any molecules of $N$ indicates that the output of the whole computation is "no", and producing any molecules of $Y$ indicates "yes". Stable: Let the output of a state be "no" if there are some $N$ molecules and no $Y$ molecules (and vice versa for "yes"). In the stable output convention, the output of the whole computation is $b \in\{$ no, yes $\}$ when the CRN reaches a state with output value $b$ from which every reachable state also has output value $b$. Limit-stable: The output of the computation is considered $b \in\{$ no, yes $\}$ when the CRN reaches a state with output $b$ and never changes it again (even though states with different output may remain reachable). The parenthetical settings have not been explicitly formalized; however the computational power indicated naturally follows by extending the results from other settings. ${ }^{3}$

Thus, surprisingly the large distinction between probability 1 and probability $<1$ computation disappears in a "limit computing" setting.

The best studied type of probability 1 computation incorporates the stable output criterion (Table 1, prob correct $=1$, stable) and was shown to be limited to semilinear predicates [1] (later extended to functions [6]). For example, consider the following CRN computing the parity predicate (a semilinear predicate). The input is encoded in the initial number of molecules of $X$, and the output is indicated by species $Y$ (yes) and $N$ (no):

$$
\begin{aligned}
& X+Y \rightarrow N \\
& X+N \rightarrow Y
\end{aligned}
$$

Starting with the input count $n \in \mathbb{N}$ of $X$, as well as $1 Y$, the CRN converges to a state where a molecule of the correct output species is present ( $Y$ if $n$ is even and $N$ if $n$ is odd) and the incorrect output species is absent. From that point on, no reaction can change the output.

Motivated by such examples, probability 1 stable computation admits the changing of output as long as the system converges with probability 1 to an output stable state - a state from which no sequence of reactions can change the output. In the above example, the states with $X$ absent are output stable. (Although the limits on stable computation were proven in the population protocols model [1] they also apply to general CRNs where infinitely many states may be reachable.)

A more stringent output convention requires irreversibly producing $N$ or $Y$ to indicate the output (i.e., " $Y$ is producible from initial state $\mathbf{x} " \Longleftrightarrow " N$ is not producible from $\mathbf{x} " \Longleftrightarrow$ $\phi(\mathbf{x})=$ yes $)$. We term such output convention committing. However, probability 1 committing

\footnotetext{
${ }^{3}$ Probability 1 committing computation: Suppose there are two inputs $\mathbf{x}_{1}, \mathbf{x}_{2} \in \mathbb{N}^{k}$ such that $\phi\left(\mathbf{x}_{1}\right)=$ no and $\phi\left(\mathbf{x}_{2}\right)=$ yes. Consider any input $\mathbf{x}_{3}$ such that $\mathbf{x}_{3} \geq \mathbf{x}_{1}$ and $\mathbf{x}_{3} \geq \mathbf{x}_{2}$. From state $\mathbf{x}_{3}$ we can produce $N$ by the sequence of reactions from $\phi\left(\mathbf{x}_{1}\right)$, and $Y$ by the sequence of reactions from $\phi\left(\mathbf{x}_{2}\right)$. Both sequences occur with some non-zero probability, and so the probability of error is non-zero for non-constant predicates. Probability $<1$ stable computation: The question of whether there is a sequence of reactions to change the output is computable and thus the stable output convention cannot give more computational power than the committing output convention. Probability < 1 limit-stable computation: Our negative result for probability 1 limit-stable computation can be modified to apply.
} 
computation is restricted to constant predicates $^{4}$ (Table 1 , prob correct $=1$, committing).

Intuitively, committing computation "knows" when it is done, while stable computation does not. Nonetheless, stable computation is not necessarily impractical. All semilinear predicates can be stably computed such that checking for output stability is equivalent to simply inspecting whether any further reaction is possible - so an outside observer can easily recognize the completion of computation [4]. While stable CRNs do not know when they are done computing, different downstream processes can be catalyzed by the $N$ and $Y$ species. As long as these processes can be undone by the presence of the opposite species, the overall computation can be in the sense stable as well. A canonical downstream process is signal amplification in which a much larger population of $\hat{N}, \hat{Y}$ is interconverted by reactions $\hat{N}+Y \rightarrow \hat{Y}+Y$ and $\hat{Y}+N \rightarrow \hat{N}+N$. Finally all semilinear predicates can be stably computed quickly (polylogarithmic in the input molecular counts).

In contrast to the limited computational abilities of the probability 1 settings just now discussed, tolerating a positive probability of error significantly expands computational power. Indeed arbitrary computable functions (Turing universal computation) can be computed with the committing output convention [16]. Turing universal computation can also be fast - the CRN simulation incurs only a polynomial slowdown. However, error is unavoidable and is due fundamentally to the inability of CRNs to deterministically detect the absence of species. (Note that Turing universal computation is only possible in CRNs when the reachable state space, i.e., molecular count, is unbounded - and is thus not meaningful in population protocols.)

When a CRN is simulating a Turing machine, errors in simulation cannot be avoided. However, can they be corrected later? In this work we develop an error correction scheme that can be applied to Turing universal computation that ensures overall output error decreases the longer the CRN runs. Indeed, in the limit of time going to infinity, with probability 1 the answer is correct.

To capture Turing-universal probability 1 computation with such an error correction process a new output convention is needed, since the committing and stable conventions are limited to much weaker forms of probability 1 computation (Table 1). Limit-stability subtly relaxes the stability requirement: instead of there being no path to change the output, we require the system to eventually stop taking such paths with probability 1 . To illustrate the difference between the original notion of stability and our notion of limit-stability, consider the reachability of the empty state (without any molecules of $Y$, in which the output is undefined) in the CRN:

$$
\begin{aligned}
& \varnothing \stackrel{1}{\rightarrow} Y \\
& Y \stackrel{1}{\rightarrow} \varnothing \\
& Y \stackrel{2}{\rightarrow} 2 Y
\end{aligned}
$$

From any reachable state, the empty state is reachable (just execute the second reaction enough times). However, with probability 1 , the empty state is visited only a finite number of times. ${ }^{5}$ If, as before, we think of the presence of $Y$ indicating a yes output, then the yes output is never stable (the empty state is always reachable), but with probability 1 a yes output will be produced and never change - i.e., it is limit-stable.

We show that with the limit-stable output convention, errors in Turing universal computation can be rectified eventually with probability 1 . Our construction is based on simulating a register

\footnotetext{
${ }^{4}$ Particulars of input encoding generally make a difference for the weaker computational settings. Indeed, probability 1 committing computation can compute more than constant predicates if they are not required to be total. For example, a committing CRN can certainly distinguish between two <-incomparable inputs.

${ }^{5}$ The second and third reactions are equivalent to the gambler's ruin problem [9], which tells us that, because the probability of increasing the count of $Y$ is twice that of decreasing its count, there is a positive probability that $Y$ 's count never reaches 0 . The first reaction can only increase this probability. Since whenever $Y$ reaches 0 , we have another try, eventually with probability 1 we will not reach 0.
} 
machine (a.k.a. Minsky counter machine) over and over in an infinite loop, increasing the number of simulated steps each time (dovetailing). Each time the CRN updates its answer to the answer given by the most recently terminated simulation. While errors occur during these simulations, our construction is designed such that with probability 1 only a finite number of errors occur (by the Borel-Cantelli Lemma), and then after some point the output will stay correct forever. The main difficulty is ensuring that errors "fail gracefully": they are allowed to cause the wrong answer to appear for a finite time, but they cannot, for instance, interfere with the dovetailing itself. Thus, although errors are unavoidable in a CRN simulation of a register machine, they can subsequently be corrected for with probability 1 . We also show that the expected time to stabilize to the correct output is polynomial in the running time of the register machine (which, however, is exponentially slower than a Turing machine).

It is natural to wonder if limit-stable probability 1 computation in CRNs characterizes exactly the computable languages. However, we show that the class of predicates limit-stable computable by CRNs with probability 1 is exactly the so-called $\Delta_{2}^{0}$ predicates (at the second level of the arithmetical hierarchy [13]). The class $\Delta_{2}^{0}$ can be characterized in terms of "limit computing" of Turing machines that can change their output a finite but unbounded number of times [15]. Thus it is not surprising that relaxing the output convention from committing to limit-stable for probability $<1$ computation increases the computational power from the computable predicates to $\Delta_{2}^{0}$. However, the key contribution of this paper is that the gap between probability $<1$ and probability 1 computation completely disappears with limit-stable output.

Note that in no way should our result be interpreted to mean that chemistry violates the Church-Turing thesis. Since we do not know when the CRN will stop changing its answer, the output of a limit-stable computation cannot be practically read out in finite time.

Relaxing the definition of probability 1 computation further does not increase computational power. An alternative definition of probability 1 limit-stable computation is to require that as time goes to infinity, the probability of expressing the correct output approaches 1 . In contrast to limit-stability, the output may change infinitely often. Note that this is exactly the distinction between almost sure convergence and convergence in probability. Our proof that probability 1 limit-stable computation is limited to $\Delta_{2}^{0}$ predicates applies to this weaker sense of convergence as well, bolstering the generality of our result. Interestingly, it is still unclear whether in CRNs there is a natural definition of probability 1 computation that exactly corresponds to the class of Turing-computable functions.

In the remainder of this paper we focus on the type of probability 1 computation captured by the notion of limit-stability, reserving the term probability 1 computation to refer specifically to probability 1 limit-stable computation.

To our knowledge, the first hints in the CRN literature of probability 1 Turing universal computation occur in ref. [19], where Zavattaro and Cardelli showed that the following question is uncomputable: Will a given CRN with probability 1 reach a state where no further reactions are possible? Although their construction relied on repeated simulations of a Turing machine, it did not use the Borel-Cantelli Lemma, and could not be directly applied to computation with output.

\section{Preliminaries}

\subsection{Computability theory}

We use the term predicate (a.k.a. language, decision problem) interchangeably to mean a subset $L \subseteq \mathbb{N}^{k}$, or equivalently a function $\phi: \mathbb{N}^{k} \rightarrow\{0,1\}$, such that $\phi(\mathbf{x})=1 \Longleftrightarrow \mathbf{x} \in L$. The halting problem is the predicate $\phi_{H}: \mathbb{N}^{2} \rightarrow\{0,1\}$ defined by $\phi_{H}(M, n)=1$ if $M(n)$ halts and $\phi_{H}(M, n)=0$ otherwise, where $M$ is a description of a Turing machine encoded as a natural number and $n \in \mathbb{N}$ 
is an input to $M$. An oracle Turing machine is a Turing machine $M$ equipped with a special "oracle" tape, with the following semantics. For an arbitrary predicate $\phi$, we write $M^{\phi}$ to denote $M$ running with oracle $\phi$. If $M^{\phi}$ writes a string representing a vector $\mathbf{q} \in \mathbb{N}^{k}$ on its oracle tape and goes to a special state $s_{\mathbf{q}}$, then in the next step, $M^{\phi}$ goes to state $s_{\phi(\mathbf{q})}$ (i.e., $s_{1}$ if $\phi(\mathbf{q})=1$ and $s_{0}$ if $\phi(\mathbf{q})=0)$. In other words, $M^{\phi}$ can determine the value $\phi(\mathbf{q})$ in constant time for any $\mathbf{q} \in \mathbb{N}^{k}$. If a predicate $\psi$ is computable by an oracle Turing machine $M$ with oracle $\phi$, then we say that $\psi$ is Turing reducible to $\phi$.

We say that a predicate $\phi: \mathbb{N}^{k} \rightarrow\{0,1\}$ is limit computable if there is a computable function $r: \mathbb{N}^{k} \times \mathbb{N} \rightarrow\{0,1\}$ such that, for all $\mathbf{x} \in \mathbb{N}^{k}, \lim _{t \rightarrow \infty} r(\mathbf{x}, t)=\phi(\mathbf{x})$. The following equivalence is known as the Shoenfield limit lemma [15].

Lemma 2.1 ( [14]). A predicate $\phi$ is limit computable if and only if it is Turing reducible to the halting problem.

We write $\Delta_{2}^{0}$ to denote the class of all limit computable predicates.

\subsection{Chemical reaction networks}

If $\Lambda$ is a finite set (in this paper, of chemical species), we write $\mathbb{N}^{\Lambda}$ to denote the set of functions $f: \Lambda \rightarrow \mathbb{N}$. Equivalently, we view an element $\mathbf{c} \in \mathbb{N}^{\Lambda}$ as a vector $\mathbf{c} \in \mathbb{N}^{|\Lambda|}$ of $|\Lambda|$ nonnegative integers, with each coordinate "labeled" by an element of $\Lambda$. Given $S \in \Lambda$ and $\mathbf{c} \in \mathbb{N}^{\Lambda}$, we refer to $\mathbf{c}(S)$ as the count of $S$ in $\mathbf{c}$. We write $\mathbf{c} \leq \mathbf{c}^{\prime}$ if $\mathbf{c}(S) \leq \mathbf{c}^{\prime}(S)$ for all $S \in \Lambda$, and $\mathbf{c}<\mathbf{c}^{\prime}$ if $\mathbf{c} \leq \mathbf{c}^{\prime}$ and $\mathbf{c} \neq \mathbf{c}^{\prime}$. Given $\mathbf{c}, \mathbf{c}^{\prime} \in \mathbb{N}^{\Lambda}$, we define the vector component-wise operations of addition $\mathbf{c}+\mathbf{c}^{\prime}$ and subtraction $\mathbf{c}-\mathbf{c}^{\prime}$. For a set $\Delta \subset \Lambda$, we view a vector $\mathbf{c} \in \mathbb{N}^{\Delta}$ equivalently as a vector $\mathbf{c} \in \mathbb{N}^{\Lambda}$ by assuming $\mathbf{c}(S)=0$ for all $S \in \Lambda \backslash \Delta$.

Given a finite set of chemical species $\Lambda$, a reaction over $\Lambda$ is a triple $\alpha=\langle\mathbf{r}, \mathbf{p}, k\rangle \in \mathbb{N}^{\Lambda} \times$ $\mathbb{N}^{\Lambda} \times \mathbb{R}^{+}$, specifying the stoichiometry (amount consumed/produced) of the reactants and products,

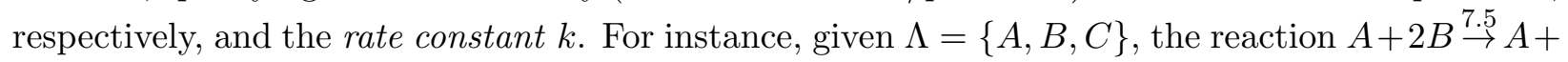
$3 C$ is represented by the triple $\langle(1,2,0),(1,0,3), 7.5\rangle$. If not specified, assume that the rate constant $k=1$. A chemical reaction network $(C R N)$ is a pair $N=(\Lambda, R)$, where $\Lambda$ is a finite set of chemical species, and $R$ is a finite set of reactions over $\Lambda$. A state of a CRN $N=(\Lambda, R)$ is a vector $\mathbf{c} \in \mathbb{N}^{\Lambda}$.

Given a state $\mathbf{c}$ and reaction $\alpha=\langle\mathbf{r}, \mathbf{p}\rangle$, we say that $\alpha$ is applicable to $\mathbf{c}$ if $\mathbf{r} \leq \mathbf{c}$ (i.e., $\mathbf{c}$ contains enough of each of the reactants for the reaction to occur). If $\alpha$ is applicable to $\mathbf{c}$, then write $\alpha(\mathbf{c})$ to denote the state $\mathbf{c}+\mathbf{p}-\mathbf{r}$ (i.e., the state that results from applying reaction $\alpha$ to $\mathbf{c})$. If $\mathbf{c}^{\prime}=\alpha(\mathbf{c})$ for some reaction $\alpha \in R$, we write $\mathbf{c} \rightarrow^{1} \mathbf{c}^{\prime}$. An execution sequence $\mathcal{E}$ is a finite or infinite sequence of states $\mathcal{E}=\left(\mathbf{c}_{0}, \mathbf{c}_{1}, \mathbf{c}_{2}, \ldots\right)$ such that, for all $i \in\{1, \ldots,|\mathcal{E}|-1\}, \mathbf{c}_{i-1} \rightarrow^{1} \mathbf{c}_{i}$. If a finite execution sequence starts with $\mathbf{c}$ and ends with $\mathbf{c}^{\prime}$, we write $\mathbf{c} \rightarrow \mathbf{c}^{\prime}$, and we say that $\mathbf{c}^{\prime}$ is reachable from $\mathbf{c}$.

\subsection{Stable decidability by CRNs}

We now review the definition of stable decidability of predicates introduced by Angluin, Aspnes, and Eisenstat [1]. Although our main theorem concerns probability 1 decidability, not stable decidability, many of the definitions of this section will be required, so it it useful to review. Intuitively, some species "vote" for a yes/no answer, and a CRN is a stable decider if it is guaranteed to reach a consensus vote that cannot change.

A chemical reaction decider $(\mathrm{CRD})$ is a tuple $\mathcal{D}=(\Lambda, R, \Sigma, \Upsilon, \phi, \mathbf{s})$, where $(\Lambda, R)$ is a $\mathrm{CRN}$, $\Sigma \subseteq \Lambda$ is the set of input species, ${ }^{6} \Upsilon \subseteq \Lambda$ is the set of voters, $\phi: \Upsilon \rightarrow\{0,1\}$ is the (Boolean) output

\footnotetext{
${ }^{6}$ In Section 3 and beyond, we restrict attention to the case that $|\Sigma|=1$, i.e., single-integer inputs. Since our main result will show that the predicates computable with probability 1 by a CRN encompass all of $\Delta_{2}^{0}$, this restriction
} 
function, and $\mathbf{s} \in \mathbb{N}^{\Lambda \backslash \Sigma}$ is the initial context. An input to $\mathcal{D}$ is a vector $\mathbf{x} \in \mathbb{N}^{\Sigma}$, or equivalently $\mathbf{x} \in \mathbb{N}^{k}$ if $|\Sigma|=k ; \mathcal{D}$ and $\mathbf{x}$ define an initial state $\mathbf{i} \in \mathbb{N}^{\Lambda}$ as $\mathbf{i}=\mathbf{s}+\mathbf{x}$ (when $\mathbf{i}$ and $\mathbf{x}$ are considered as elements of $\left.\mathbb{N}^{\Lambda}\right){ }^{7}$ When $\mathbf{i}$ is clear from context, we say that a state $\mathbf{c}$ is reachable if $\mathbf{i} \rightarrow \mathbf{c}$.

We extend $\phi$ to a partial function $\Phi: \mathbb{N}^{\Lambda} \rightarrow\{0,1\}$ as follows. $\Phi(\mathbf{c})$ is undefined if either $\mathbf{c}(V)=0$ for all $V \in \Upsilon$, or if there exist $N, Y \in \Upsilon$ such that $\mathbf{c}(N)>0, \mathbf{c}(Y)>0, \phi(N)=0$ and $\phi(Y)=1$. Otherwise, $(\exists b \in\{0,1\})(\forall V \in \Upsilon)(\mathbf{c}(V)>0 \Longrightarrow \phi(V)=b)$; in this case, the output $\Phi(\mathbf{c})$ of state $\mathbf{c}$ is $b$. In other words $\Phi(\mathbf{c})=b$ if some voters are present and they all vote $b$.

If $\Phi(\mathbf{y})$ is defined and every state $\mathbf{y}^{\prime}$ reachable from $\mathbf{y}$ satisfies $\Phi(\mathbf{y})=\Phi\left(\mathbf{y}^{\prime}\right)$, then we say that $\mathbf{y}$ is output stable, i.e., if $\mathbf{y}$ is ever reached, then no reactions can ever change the output. We say that $\mathcal{D}$ stably decides the predicate $\phi: \mathbb{N}^{k} \rightarrow\{0,1\}$ if, for all input states $\mathbf{x} \in \mathbb{N}^{k}$, for every state $\mathbf{c}$ reachable from $\mathbf{x}$, there is an output stable state $\mathbf{y}$ reachable from $\mathbf{c}$ such that $\Phi(\mathbf{y})=\phi(\mathbf{x})$. In other words, no sequence of reactions (reaching state $\mathbf{c}$ ) can prevent the CRN from being able to reach the correct answer (since $\mathbf{c} \rightarrow \mathbf{y}$ and $\Phi(\mathbf{y})=\phi(\mathbf{x})$ ) and staying there if reached (since $\mathbf{y}$ is output stable).

At first glance, this definition appears too weak to claim that the CRN is "guaranteed" to reach the correct answer. It merely states that the CRN is guaranteed stay in states from which it is possible to reach the correct answer. If the set of states reachable from the initial state $\mathbf{x}$ is finite (as with all population protocols since the total molecular count is bounded), however, then it is easy to show that with probability 1 , the CRN will eventually reach an output stable state with the correct answer, assuming the system's evolution is governed by stochastic chemical kinetics, defined later.

Why is this true? By the definition of stable decidability, for all reachable states $\mathbf{c}$, there exists a reaction sequence $r_{\mathbf{c}}$ such that some output stable state $\mathbf{y}$ is reached after applying $r_{\mathbf{c}}$ to $\mathbf{c}$. If there are a finite number of reachable states, then there exists $\epsilon>0$ such that for all reachable states $\mathbf{c}, \operatorname{Pr}\left[r_{\mathbf{c}}\right.$ occurs upon reaching $\left.\mathbf{c}\right] \geq \epsilon$. For any such state $\mathbf{c}$ visited $\ell$ times, the probability that $r_{\mathbf{c}}$ is not followed after all $\ell$ visits is at most $(1-\epsilon)^{\ell}$, which approaches 0 as $\ell \rightarrow \infty$. Since some state $\mathbf{c}$ must be visited infinitely often in a reaction sequence that avoids an output stable state $\mathbf{y}$ forever, this implies that the probability is 0 that $r_{\mathbf{c}}$ is never followed after reaching $\mathbf{c}$. Conversely, if a correct output-stable state is reached with probability 1 , then this implies a correct output-stable state is always reachable, even with an infinite reachable state space, because if a reachable state $\mathbf{c}$ existed from which no correct output-stable state was reachable, then since $\mathbf{c}$ is reached with positive probability, the CRN would have probability strictly less than 1 to reach a correct output stable state.

\subsection{Probability 1 decidability by CRNs}

In order to define probability 1 computation with CRNs, we first review the model of stochastic chemical kinetics. It is widely used in quantitative biology and other fields dealing with chemical reactions between species present in small counts [10]. It ascribes probabilities to execution sequences, and also defines the time of reactions.

A reaction is unimolecular if it has one reactant and bimolecular if it has two reactants. We use no higher-order reactions in this paper.

will not be crucial, since any computable encoding function $e: \mathbb{N}^{k} \rightarrow \mathbb{N}$ that represents $k$-tuples of integers as a single integer, and its inverse decoding function $d: \mathbb{N} \rightarrow \mathbb{N}^{k}$, can be computed by the CRN to handle a $k$-tuples of inputs that is encoded into the count of a single input species $X$. Such encoding functions are provably not computable by semilinear functions, so this distinction is more crucial in the realm of stable computation by CRDs $[2,6]$.

${ }^{7}$ In other words, species in $\Lambda \backslash \Sigma$ must always start with the same counts, and counts of species in $\Sigma$ are varied to represent different inputs to $\mathcal{D}$, similarly to a Turing machine that starts with different binary string inputs, but the Turing machine must always start with the same initial state and tape head position. 
The kinetics of a CRN is described by a continuous-time Markov process as follows. The system has some volume $v \in \mathbb{R}^{+}$that affects the transition rates, which may be fixed or allowed to vary over time; in this paper we assume a constant volume of $1 .^{8}$ In state $\mathbf{c}$, the propensity of a unimolecular reaction $\alpha: X \stackrel{k}{\rightarrow} \ldots$ in state $\mathbf{c}$ is $\rho(\mathbf{c}, \alpha)=k \cdot \mathbf{c}(X)$. The propensity of a bimolecular reaction $\alpha: X+Y \stackrel{k}{\rightarrow} \ldots$, where $X \neq Y$, is $\rho(\mathbf{c}, \alpha)=k \cdot \frac{\mathbf{c}(X) \cdot \mathbf{c}(Y)}{v}$. The propensity of a bimolecular reaction $\alpha: X+X \stackrel{k}{\rightarrow} \ldots$ is $\rho(\mathbf{c}, \alpha)=\frac{k}{2} \cdot \frac{\mathbf{c}(X) \cdot(\mathbf{c}(X)-1)}{v}$. The propensity function governs the evolution of the system as follows. The time until the next reaction occurs is an exponential random variable with rate $\rho(\mathbf{c})=\sum_{\alpha \in R} \rho(\mathbf{c}, \alpha)$ (note $\rho(\mathbf{c})=0$ if and only if no reactions are applicable to $\mathbf{c}$ ). The probability that next reaction will be a particular $\alpha_{\text {next }}$ is $\frac{\rho\left(\mathbf{c}, \alpha_{\text {next }}\right)}{\rho(\mathbf{c})}$.

We now define probability 1 computation by CRDs. Our definition is based on the limit-stable output convention as discussed in the introduction. Let $\mathcal{D}=(\Lambda, R, \Sigma, \Upsilon, \phi, \mathbf{s})$ be a CRD. Let $\mathcal{E}=\left(\mathbf{c}_{0}, \mathbf{c}_{1}, \ldots\right)$ be an execution sequence of $\mathcal{D}$. In general $\mathcal{E}$ could be finite or infinite, depending on whether $\mathcal{D}$ can reach a terminal state (one in which no reaction is applicable); however, in this paper all CRDs will have no reachable terminal states, so assume $\mathcal{E}$ is infinite. We say that $\mathcal{E}$ has a defined output if there exists $b \in\{0,1\}$ such that, for all but finitely many $i \in \mathbb{N}, \Phi\left(\mathbf{c}_{i}\right)=b$. In other words, $\mathcal{E}$ eventually stabilizes to a certain answer. In this case, write $\Phi(\mathcal{E})=b$; otherwise, let $\Phi(\mathcal{E})$ be undefined.

If $\mathbf{x} \in \mathbb{N}^{k}$, write $\mathcal{E}(\mathcal{D}, \mathbf{x})$ to denote the random variable representing an execution sequence of $\mathcal{D}$ on input $\mathbf{x}$, resulting from the Markov process described previously. We say that $\mathcal{D}$ decides $\phi: \mathbb{N}^{k} \rightarrow\{0,1\}$ with probability 1 if, for all $\mathbf{x} \in \mathbb{N}^{k}$,

$$
\operatorname{Pr}[\Phi(\mathcal{E}(\mathcal{D}, \mathbf{x}))=\phi(\mathbf{x})]=1
$$

\section{Turing-decidable predicates}

This section describes how a CRD can decide an arbitrary Turing-decidable predicate with probability 1 . This construction also contains most of the technical details needed to prove our positive result that CRDs can decide arbitrary $\Delta_{2}^{0}$ predicates with probability 1 . The proof is via simulation of register machines, which are able to simulate arbitrary Turing machines if at least 3 registers are used. This section describes the simulation and gives intuition for how it works. Section 4 proves its correctness (proof sketch in this version of the paper). Section 5 shows how to extend the construction to handle $\Delta_{2}^{0}$ predicates and prove that no more predicates can be decided with probability 1 by a CRD.

\subsection{Register machines}

A register machine $M$ has $m$ registers $r_{1}, \ldots, r_{m}$ that can each hold a non-negative integer. $M$ is programmed by a finite sequence (lines) of instructions. There are four types of instructions: accept, reject, inc $\left(r_{j}\right)$, and $\operatorname{dec}\left(r_{j}, k\right)$. For simplicity, we describe our construction for singleinput register machines and thus predicates $\phi: \mathbb{N} \rightarrow\{0,1\}$, but it can be easily extended to more inputs. The input $n \in \mathbb{N}$ to $M$ is the initial value of register $r_{1}$, and the remaining $m-1$ registers

\footnotetext{
${ }^{8} \mathrm{~A}$ common restriction is to assume the finite density constraint, which stipulates that arbitrarily large mass cannot occupy a fixed volume, and thus the volume must grow proportionally with the total molecular count. With some minor modifications to ensure relative rates of reactions stay the same (even though all bimolecular reactions would be slowed down in absolute terms), our construction would work under this assumption, although the time analysis would change. For the sake of conceptual clarity, we present the construction assuming a constant volume. The issue is discussed in more detail in Section 4.
} 
are used to perform a computation on the input, which by convention are set to 0 initially. The semantics of execution of $M$ is as follows. The initial line is the first instruction in the sequence. If the current line is accept or reject, then $M$ halts and accepts or rejects, respectively. If the current line is inc $\left(r_{j}\right)$, then register $r_{j}$ is incremented, and the next line in the sequence becomes the current line. If the current line is $\operatorname{dec}\left(r_{j}, k\right)$, then register $r_{j}$ is decremented, and the next line in the sequence becomes the current line, unless $r_{j}=0$, in which case it is left at 0 and line $k$ becomes the current line. In other words, $M$ executes a straight-line program, with a "conditional jump" that occurs when attempting to decrement a 0-valued register. For convenience we assume there is a fifth type of instruction goto $(k)$, meaning "unconditionally jump to line $k$ ". This can be indirectly implemented by decrementing a special register $r_{0}$ that always has value 0 , or easily implemented in a CRN directly. The set of input values $n$ that cause the machine to accept is then the language/predicate decided by the machine. For example, the following register machine decides the parity of the initial value of register $r_{1}$ :

$\begin{array}{ll}\text { 1: } & \operatorname{dec}\left(r_{1}, 5\right) \\ \text { 2: } & \operatorname{dec}\left(r_{1}, 4\right) \\ \text { 3: } & \text { goto }(1) \\ \text { 4: } & \text { accept } \\ \text { 5: } & \text { reject }\end{array}$

Chemical reaction networks can be used to simulate any register machine through a simple yet error-prone construction, which is similar to the simulation described in [16]. We now describe the simulation and highlight the source of error. Although this simulation may be error-prone, the effect of the errors has a special structure, and our main construction will take advantage of this structure to keep errors from invalidating the entire computation. Specifically, there is a possibility of an error precisely when the register machine performs a conditional jump. We highlight this fact in the construction below to motivate the modifications we make to the register machine in Section 3.2 .

For a register machine with $l$ lines of instructions and $m$ registers, create molecular species $L_{1}, \ldots, L_{l}$ and $R_{1}, \ldots, R_{m}$. The presence of molecule $L_{i}$ is used to indicate that the register machine is in line $i$. Since the register machine can only be in one line at a time, there will be exactly one molecule of the form $L_{i}$ present in the solution at any time. The count of species $R_{j}$ represents the current value of register $r_{j}$. The following table shows the reactions to simulate an instruction of the register machine, assuming the instruction occurs on line $i$ :

\begin{tabular}{|l|l|}
\hline accept & $L_{i} \rightarrow H_{Y}$ \\
\hline reject & $L_{i} \rightarrow H_{N}$ \\
\hline goto $(k)$ & $L_{i} \rightarrow L_{k}$ \\
\hline inc $\left(r_{j}\right)$ & $L_{i} \rightarrow L_{i+1}+R_{j}$ \\
\hline $\operatorname{dec}\left(r_{j}, k\right)$ & $L_{i}+R_{j} \rightarrow L_{i+1}$ \\
& $L_{i} \rightarrow L_{k}$ \\
\hline
\end{tabular}

The first four reactions are error-free simulations of the corresponding instructions. The final two reactions are an error-prone way to decrement register $r_{j}$. If $r_{j}=0$, then only the latter reaction is possible, and when it occurs it is a correct simulation of the instruction. However, if $r_{j}>0$ (hence there are a positive number $R_{j}$ molecules in solution), then either reaction is possible. While only the former reaction is correct, the latter reaction could still occur. The semantic effect this has on the register machine being simulated is that, when a decrement $\operatorname{dec}\left(r_{j}, k\right)$ is possible because $r_{j}>0$, the machine may nondeterministically jump to line $k$ anyway. Our two goals in the subsequently described construction are 1) to reduce sufficiently the probability of this error occurring each time a decrement instruction is executed so that with probability 1 errors eventually 
stop occurring, and 2) to set up the simulation carefully so that it may recover from any finite number of errors.

\subsection{Simulating register machine}

We will first describe how to modify $M$ to obtain another register machine $S$ which is easier for the CRD to simulate repeatedly to correct errors. There are two general modifications we make to $M$ to generate $S$. The first consists of adding several instructions before $M$ 's first line and at the end (denoted line $h$ below). The second consists of adding a pair of instructions before every decrement of $M$. We now describe these modifications in more detail.

Intuitively, $S$ maintains a bound $b \in \mathbb{N}$ on the total number of decrements $M$ is allowed to perform, and $S$ halts if $M$ exceeds this bound. ${ }^{9}$ Although $M$ halts if no errors occur, an erroneous jump may take $M$ to a configuration unreachable from the initial configuration, so that $M$ would not halt even if simulated correctly from that point on. To ensure that $S$ always halts, it stores $b$ in such a way that errors cannot corrupt its value. $S$ similarly stores $M$ 's input $n$ in an incorruptible way. Since we know in advance that $M$ halts on input $n$ but do not know how many steps it will take, the CRD will simulate $S$ over and over again on the same input, each time incrementing the bound $b$, so that eventually $b$ is large enough to allow a complete simulation of $M$ on input $n$, assuming no errors.

If the original register machine $M$ has $m$ registers $r_{1}, \ldots, r_{m}$, then the simulating register machine $S$ will have $m+4$ registers: $r_{1}, \ldots, r_{m}, r_{\mathrm{in}}, r_{\mathrm{in}^{\prime}}, r_{t}, r_{t^{\prime}}$. The first $m$ registers behave exactly as in $M$, and the additional 4 registers will be used to help $S$ maintain the input $n$ and bound $b$.

The registers $r_{\text {in }}$ and $r_{\text {in }}$ are used to store the input value $n$. The input value $n$ is given to $S$, and initially stored in $r_{\mathrm{in}}$, and passed to $r_{\mathrm{in}}$ and $r_{1}$ before any other commands are executed. This additional step still allows the input $n$ to be used in register $r_{1}$, while retaining the input value $n$ in $r_{\mathrm{in}}$ for the next run of $S$. We want to enforce the invariant that, even if errors occur, $r_{\mathrm{in}}+r_{\mathrm{in}^{\prime}}=n$, so that the value $n$ can be restored to register $r_{\text {in }}$ when $S$ is restarted. To ensure that this invariant is maintained, the values of registers $r_{\mathrm{in}}$ and $r_{\mathrm{in}}$ change only with one of the two following sequences: $\operatorname{dec}\left(r_{\mathrm{in}}, k\right) ; \operatorname{inc}\left(r_{\mathrm{in}^{\prime}}\right)$ or $\operatorname{dec}\left(r_{\mathrm{in}^{\prime}}, k\right) ; \operatorname{inc}\left(r_{\mathrm{in}}\right)$ (it is crucial that the decrement comes before the increment, so that the increment is performed only if the decrement is successful). The only time the invariant $r_{\mathrm{in}}+r_{\mathrm{in}^{\prime}}=n$ is not maintained is between the decrement and the subsequent increment. However, once the decrement is successfully performed, there is no possibility of error in the increment, so the invariant is guaranteed to be restored.

Registers $r_{t}$ and $r_{t^{\prime}}$ are used to record and bound the number of decrements performed by $M$, and their values are modified similarly to $r_{\text {in }}$ and $r_{\mathrm{in}}$ so that $S$ maintains the invariant $r_{t}+r_{t^{\prime}}=b$ throughout each execution. The following lines are inserted before every decrement of $M$ (assuming the decrement of $M$ occurs on line $i+2)$ :

$$
\begin{aligned}
i: & \operatorname{dec}\left(r_{t}, h\right) \\
i+1: & \operatorname{inc}\left(r_{t^{\prime}}\right)
\end{aligned}
$$

$S$ uses lines $h$ and $h+1$ added at the end to halt if $M$ exceeds the decrement bound:

$$
\begin{aligned}
h: & \text { inc }\left(r_{t}\right) \\
h+1: & \text { reject }
\end{aligned}
$$

As $S$ performs each decrement command in $M, r_{t}$ is decremented and $r_{t^{\prime}}$ is incremented. If the value of $r_{t}$ is zero, then this means $M$ has exceeded the allowable number of decrements, and

\footnotetext{
${ }^{9}$ It is sufficient to bound the number of decrements, rather than total instructions, since we may assume without loss of generality that $M$ contains no "all-increment" cycles. (If it does then either these lines are not reachable or $M$ enters an infinite loop.) Thus any infinite computation of $M$ must decrement infinitely often.
} 
computation halts (with a reject, chosen merely as a convention) immediately after incrementing $r_{t}$ on line $h$. This final increment ensures that when the CRD simulates $S$ again, the bound $b=r_{t}+r_{t^{\prime}}$ will be 1 larger than it was during the previous run of $S$.

$S$ is simulated multiple times by the CRD. To make it easier for the CRD to "reset" $S$ by simply setting the current instruction to be the first line, $S$ assumes that the work registers $r_{2}, \ldots, r_{m}$ are initially positive and must be set to 0 . It sets $r_{1}$ to 0 before using $r_{\text {in }}$ and $r_{\text {in }}$ to initialize $r_{1}$ to $n$. In both pairs of registers $r_{\mathrm{in}}, r_{\mathrm{in}^{\prime}}$ and $r_{t}, r_{t^{\prime}}, S$ sometimes needs to transfer the entire quantity stored in one register to the other. We describe below a "macro" for this operation, which we call flush. The following three commands will constitute the flush $\left(r_{j}, r_{j^{\prime}}\right)$ command for any registers $r_{j}$ and $r_{j^{\prime}}$.

\begin{tabular}{|l|rl|}
\hline $\operatorname{flush}\left(r_{j}, r_{j^{\prime}}\right)$ & $i:$ & $\operatorname{dec}\left(r_{j}, i+3\right)$ \\
& $i+1:$ & $\operatorname{inc}\left(r_{j^{\prime}}\right)$ \\
& $i+2:$ & $\operatorname{goto}(i)$ \\
\hline
\end{tabular}

While $r_{j}$ has a non-zero value, it will be decremented and $r_{j^{\prime}}$ will be incremented. To flush into more than one register, we can increment multiple registers in the place of line $i+1$ above. We denote this as $\operatorname{flush}\left(r_{j}, r_{j^{\prime}}\right.$ and $\left.r_{j^{\prime \prime}}\right)$.

To set register $r_{j}$ to 0 , we use the following macro, denoted empty $\left(r_{j}\right)$.

\begin{tabular}{|c|c|}
\hline empty $\left(r_{j}\right)$ & $\begin{aligned} i: & \operatorname{dec}\left(r_{j}, i+2\right) \\
i+1: & \operatorname{goto}(i)\end{aligned}$ \\
\hline
\end{tabular}

If an error occurs in a flush, the invariant on the sum of the two registers will still be maintained. If an error occurs on an empty, the effect will be that the register maintains a positive value. Both effects can be remedied by an error-free repeat of the same macro.

Combining all techniques described above, the first instructions of $S$ when simulating $M$ will be as follows:

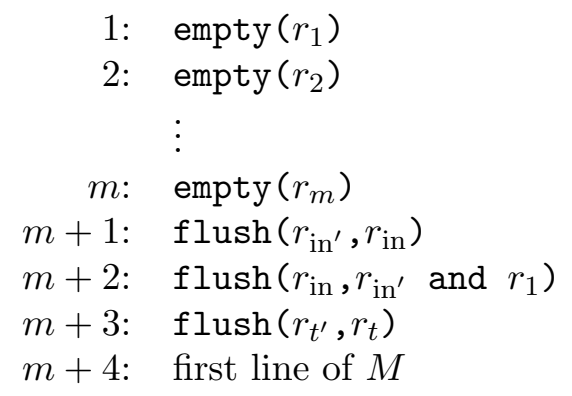

The first $m$ lines ensure that registers $r_{1}, \ldots, r_{m}$ are initialized to zero. Lines $m+1$ and $m+2$ pass the input value $n$ (stored as $r_{\mathrm{in}}+r_{\mathrm{in}}{ }^{\prime}$ ) to register $r_{1}$ (input register of $M$ ) while still saving the input value in $r_{\mathrm{in}}$ to be reused the next time $S$ is run. Line $m+3$ passes the full value of $r_{t^{\prime}}$ to $r_{t}$, so that the value of register $r_{t}$ can be used to bound the number of jumps in the register machine. After line $m+3, S$ executes the commands of $M$, starting with either the initial line of $M$ (or decrementing $r_{t}$ if the initial line of $M$ is a decrement command as explained above).

Let $d_{n}$ be the number of decrements $M$ makes on input $n$ without errors. If $S$ is run without errors from an initial configuration (starting on line 1) with $r_{\mathrm{in}}+r_{\text {in }^{\prime}}^{\prime}=n$ and $r_{t}+r_{t^{\prime}} \geq d_{n}$, then it will successfully simulate $M$. Since the invariants on $r_{\mathrm{in}}+r_{\mathrm{in}^{\prime}}=n$ and $r_{t}+r_{t^{\prime}}$ are maintained throughout the computation - even in the face of errors - the only thing required to reset $S$ after it halts is to set the current line to 1 . 


\subsection{CRD simulation of the modified register machine}

We now construct a $\mathrm{CRD} \mathcal{D}$ to simulate $S$, while reducing the probability of an error each time an error could potentially occur. Besides the species described in Section 3.1, we introduce several new species: voting species $N$ and $Y$, an "accuracy" species $A$, and four "clock" species $C_{1}, C_{2}, C_{3}$, and $C_{4}$. The accuracy and clock species will be used to reduce the probability of an error occurring. The initial state of $\mathcal{D}$ on input $n \in \mathbb{N}$ is $\left\{n R_{\text {in }}, 1 L_{1}, 1 Y, 1 C_{1}\right\}$ - i.e., start with register $r_{\text {in }}=n$, initialize the register machine $S$ at line 1, have initial vote "yes" (arbitrary choice), and start the "clock module" in the first stage.

Recall that the only source of error in the CRD simulation is from the decrement command $\operatorname{dec}\left(r_{j}, h\right)$, when $R_{j}$ is present, but the jump reaction $L_{i} \rightarrow L_{k}$ occurs instead of the decrement reaction $L_{i}+R_{j} \rightarrow L_{i+1}$. This would cause the CRD to erroneously perform a jump when it should instead decrement register $r_{j}$. To decrease the probability of this occurring, we can slow down the jump reaction, thus decreasing the probability of it occurring before the decrement reaction when $R_{j}$ is present.

The following reactions we call the "clock module," which implement a random walk that is biased in the reverse direction, so that $C_{4}$ is present sporadically, with the bias controlling the frequency of time $C_{4}$ is present. The count of "accuracy species" $A$ controls the bias:

$$
\begin{array}{ll}
C_{1} \rightarrow C_{2}, & C_{2}+A \rightarrow C_{1}+A, \\
C_{2} \rightarrow C_{3}, & C_{3}+A \rightarrow C_{2}+A, \\
C_{3} \rightarrow C_{4}, & C_{4}+A \rightarrow C_{3}+A .
\end{array}
$$

We modify the conditional jump reaction to require a molecule of $C_{4}$ as a reactant, as shown below. Increasing the count of species $A$ decreases the expected time until the reaction $C_{i+1}+A \rightarrow$ $C_{i}+A$ occurs, while leaving the expected time until reaction $C_{i} \rightarrow C_{i+1}$ constant. This has the effect that $C_{4}$ is present less frequently (hence the conditional jump reaction is slowed). Intuitively, with an $\ell$-stage clock, if there are $a$ molecules of $A$, the frequency of time that $C_{\ell}$ is present is less than $\frac{1}{a^{\ell-1}}$. A stage $\ell=4$ clock is used to ensure that the error decreases quickly enough that with probability 1 a finite number of errors are ever made, and the last error occurs in finite expected time. A more complete analysis of the clock module is contained in Section 4.

To use the clock module to make decrement instructions unlikely to incur errors, we change the CRD simulation of a decrement command to be the following two reactions:

$$
\begin{array}{|l|l|}
\hline \operatorname{dec}\left(r_{j}, k\right) & L_{i}+R_{j} \rightarrow L_{i+1}+A \\
& L_{i}+C_{4} \rightarrow L_{k}+C_{1}+A \\
\hline
\end{array}
$$

The jump reaction produces a $C_{1}$ molecule so the clock can be restarted for the next decrement command. Both reactions produce an additional $A$ molecule to increase the accuracy of the next decrement command. As we continue to perform decrements, the random walk from $C_{1}$ to $C_{4}$ acquires a stronger reverse bias, so the conditional jump becomes less likely to occur erroneously.

The accept, reject, goto, and inc commands cannot result in errors for the CRD simulation, so we keep their reactions unchanged from Section 3.1. Assuming the command occurs on line $i$, the reactions to simulate each command are given in the following table:

\begin{tabular}{|l|l|}
\hline accept & $L_{i} \rightarrow H_{Y}$ \\
\hline reject & $L_{i} \rightarrow H_{N}$ \\
\hline goto $(k)$ & $L_{i} \rightarrow L_{k}$ \\
\hline $\operatorname{inc}\left(r_{j}\right)$ & $L_{i} \rightarrow L_{i+1}+R_{j}$ \\
\hline
\end{tabular}

After the CRD has completed the simulation and stabilizes, we would like the CRD to store the output of computation (either $H_{Y}$ or $H_{N}$ ) and restart. At any time, there is precisely one molecule 
of either of the two voting species $Y$ or $N$ and none of the other, representing the CRD's "current vote." The vote is updated after each simulation of $S$, and $S$ is reset to its initial configuration, via these reactions:

$$
\begin{aligned}
& H_{Y}+Y \rightarrow L_{1}+Y \\
& H_{Y}+N \rightarrow L_{1}+Y \\
& H_{N}+Y \rightarrow L_{1}+N \\
& H_{N}+N \rightarrow L_{1}+N
\end{aligned}
$$

In Section 4, we prove that with probability 1, this CRD decides the predicate decided by $M$.

\subsection{Example of construction of CRD from register machine}

In this section we give an example of a register machine $M$ that decides a predicate, a register machine $S$ that simulates $M$ as described in Section 3.2, and a CRD $\mathcal{D}$ that simulates $S$. In this example $M$ decides the parity of input $n$.

The machine $M$ requires only the input register $r_{1}$. Register machine $M$ can be simulated by register machine $S$ allowing $b$ decrements, where the CRN increments $b$ each time it simulates $S$. Notice that with this bound on the number of jumps, $S$ will successfully simulate $M$ if $b$ is at least the number of decrements made by $M$ on input $n$. Machine $S$ has 5 registers: $r_{1}, r, r^{\prime}, t$, and $t^{\prime}$, and extra commands as described in Section 3.3. Finally, machine $S$ can be simulated by a CRD $\mathcal{D}$ with reactions as described in Section 3.3. All three constructions for the example predicate are shown side-by-side in the following table. The commands in $M$ are vertically aligned with their respective representations in $S$ and the CRN. The initial state of $\mathcal{D}$ is $\left\{1 L_{1}, 1 Y, 1 C_{1}, n R\right\}$.

In addition to reactions in the table, $\mathcal{D}$ requires the clock module reactions:

$$
\begin{array}{ll}
C_{1} \rightarrow C_{2}, & C_{2}+A \rightarrow C_{1}+A, \\
C_{2} \rightarrow C_{3}, & C_{3}+A \rightarrow C_{2}+A, \\
C_{3} \rightarrow C_{4}, & C_{4}+A \rightarrow C_{3}+A
\end{array}
$$




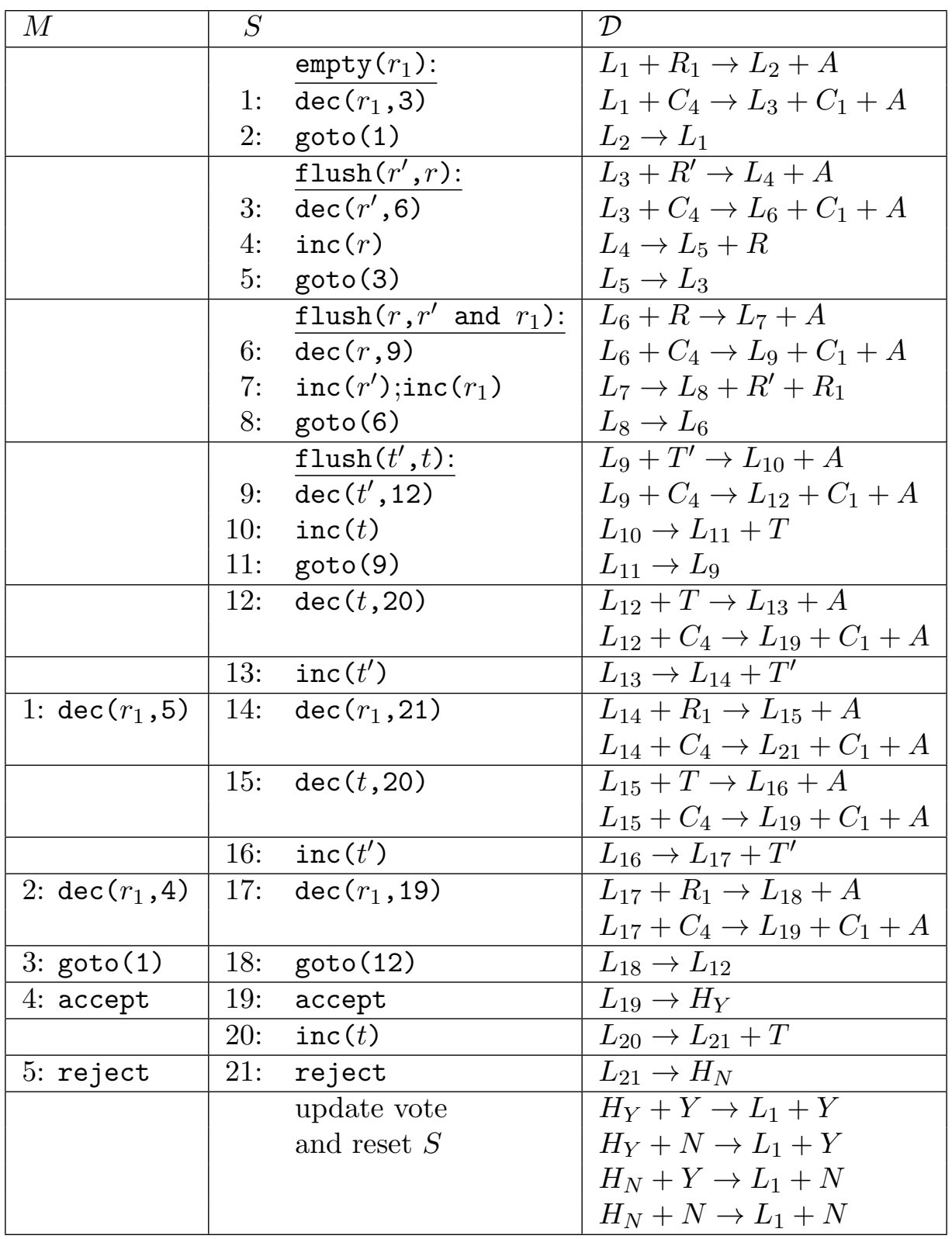

\section{Correctness of simulation}

In this section we show that the construction of Section 3 is correct.

Theorem 4.1. Let $\mathcal{D}$ be the $C R D$ described in Section 3, simulating an arbitrary register machine $M$ deciding predicate $\phi: \mathbb{N} \rightarrow\{0,1\}$. Then $\mathcal{D}$ decides $\phi$ with probability 1 . Furthermore, the expected time until $\mathcal{D}$ stabilizes to the correct answer is $O\left(t_{n}^{8}\right)$, where $t_{n}$ is the number of steps $M$ takes to halt on input $n$.

Proof. We will show three things. First, so long as $S$ is repeatedly simulated by $\mathcal{D}$, if a finite number of errors occur, then eventually it correctly simulates $M$ (and therefore $\mathcal{D}$ 's voters eventually are updated to the correct value and stay there). Second, with probability $1 \mathcal{D}$ makes only a finite number of errors in simulating $S$. Finally, we bound the expected time to stabilize to the correct answer. 
Let $n \in \mathbb{N}$ be the input to $M$. We note that the CRD simulation of $S$ may err in the simulation, but the errors take a very particular form. If $\mathcal{D}$ makes an error, it is because the following two reactions (corresponding to the two branches a decrement instruction might follow) are possible, but executing the second is an error:

$$
\begin{aligned}
& L_{i}+R_{j} \rightarrow L_{i+1}+A \\
& L_{i}+C_{4} \rightarrow L_{k}+C_{1}+A
\end{aligned}
$$

(This applies similarly if the decrement is on registers $r, r^{\prime}, t$, or $t^{\prime}$.) This corresponds to the command "in line $i$, decrement register $j$ and go to line $i+1$, unless the register is 0 , in which case go to line $k$ instead of $i+1$." The error in simulation can be captured by a modified model of a register machine in which the following error may occur: when doing this decrement instruction, even if register $j$ is positive, it is possible for the machine to nondeterministically choose to jump to line $k$ without decrementing register $j$.

Even in the face of such errors, $S$ maintains the following useful invariants.

1. $r+r^{\prime}=n$ (except immediately after decrementing $r$ but before incrementing $r^{\prime}$, when the sum is $n-1$, but since increments cannot have errors, the sum is guaranteed to be immediately restored to $n$ ).

2. If $S$ has been simulated by $\mathcal{D}$ several times, and in $N$ of these simulations, $S$ 's simulation of $M$ has "timed out" (the register $t$ decremented to 0 before the simulation of $M$ was complete), then on the next simulation of $S$, we will have $t+t^{\prime}=N+1$. As before, this invariant is only violated in between decrementing $t$ and incrementing $t^{\prime}$, but since the subsequent increment is error-free, the invariant $t+t^{\prime}=N+1$ is immediately restored.

The above are easy to check by inspection of the machine $S$.

Since the initial instructions of $S$ (assuming they are executed without error) reset registers $r$, $t^{\prime}$, and $r_{2}, \ldots, r_{m}$ to 0 , and they reset $r_{1}$ to be its correct initial value $n$, any configuration of $S$ such that

1. the current line is the first,

2. $r+r^{\prime}=n$, and

3. $t+t^{\prime}$ is at least the total number of decrements $M$ will do on input $n$

is a correct initial configuration. Since $S$ maintains (2) and (3) even in the face of errors, this means that to reset $S, \mathcal{D}$ needs only to consume the "halt molecule" $H_{Y}$ or $H_{N}$ and produce $L_{1}$, which is precisely what is done by reactions (3.1), (3.2), (3.3), and (3.4).

Therefore, if $\mathcal{D}$ eventually stops making errors in simulating $S$, then $S$ faithfully simulates $M$ (from some configuration possibly unreachable from the input), for at most $t+t^{\prime}$ decrements in $M$, at which point $\mathcal{D}$ resets $S$. At that point, $\mathcal{D}$ simulates $S$ repeatedly, and after each time that $S$ stops its simulation of $M$ because the $t$ register is depleted, the sum $t+t^{\prime}$ is incremented. Therefore the sum $t+t^{\prime}$ eventually becomes large enough for $S$ to simulate $M$ completely. At that point the voters are updated to the correct value and stay there forever.

It remains to show that $\mathcal{D}$ is guaranteed to make a finite number of errors in simulating $S$. Recall that the only error possible is, on a decrement instruction, to execute a "jump" even when a register is positive. Each time that a decrement instruction is reached, the count of molecule $A$ is incremented (whether the decrement or the jump occurs). The "clock" module implements a biased random walk on the state space $\left\{C_{1}, C_{2}, C_{3}, C_{4}\right\}$ with rate 1 to increment the subscript and rate equal to the count of $A$ - denoted by $\ell$ - to decrement the index. If this Markov process is 
in equilibrium, the probability of the process being in state $C_{4}$ is $\frac{1}{\ell^{3}}$ (see [16] for example). Since the process starts in state $C_{1}$, every nonequilibrium distribution reached at any time after starting assigns lesser probability to state $C_{4}$, so the probability of the process being in state $C_{4}$ is at most $\frac{1}{\ell^{3}}$.

Thus, if there is at least one $R_{j}$ molecule, the probability that $L_{i}$ encounters $C_{4}$ prior to encountering $R_{j}$ is at most $\frac{1}{\ell^{3}}$. At each decrement instruction (whether the decrement or the jump happens), $A$ 's count is incremented, so for all $\ell \in \mathbb{Z}^{+}$, the probability of an error on the $\ell^{\prime}$ th decrement instruction (counting from the beginning of the entire execution of $\mathcal{D}$; not just from the beginning of one simulation of $S$ ) is at most $\frac{1}{\ell^{3}}$. Since $\sum_{\ell=1}^{\infty} \frac{1}{\ell^{3}}<\infty$, by the Borel-Cantelli lemma, the probability that only finitely many errors occur is 1. Recall that the Borel-Cantelli lemma does not require the events to be independent, and indeed they are not in our case (e.g., failing to decrement may take the register machine to a line in which the next attempted decrement is more or less likely to occur than if it had gone to the correct line).

In fact, error probability $\frac{1}{\ell^{2}}$ would have sufficed in the previous argument, but the lower error probability of $\frac{1}{\ell^{3}}$ will help us derive our expected time until errors cease.

We first establish a bound on the expected total number of steps (of the simulated register machine), and then use this to bound the expected time. For each $\ell$, the probability that the last error occurs on the $\ell^{\prime}$ th attempted decrement is at most $\frac{1}{\ell^{3}} \prod_{j=\ell+1}^{\infty}\left(1-\frac{1}{j^{3}}\right)$. Thus the expected step (measured over all attempted decrements, not over all steps of the register machine) of the last error is at most

$$
\sum_{\ell=1}^{\infty} \ell \cdot \frac{1}{\ell^{3}} \prod_{j=\ell+1}^{\infty}\left(1-\frac{1}{j^{3}}\right)<\sum_{\ell=1}^{\infty} \frac{1}{\ell^{2}}=\frac{\pi^{2}}{6} .
$$

Hence we have only a constant expected number of errors.

Because of this, the expected steps to stabilization is at most $t_{e}+t_{t}+t_{n}$, where $t_{e}=O(1)$ is the expected number of simulated steps until the last error happens, $t_{n}$ is the expected number of steps for one simulation of the register machine $M$ to complete, given input $n$ and assuming no errors happen, and $t_{t}$ is the expected number of steps until the timer $T$ reaches to at least $t_{n}$ the number of steps required for the register machine to halt on input $n$. Since the timer increases by 1 each simulation, $t_{t}=O\left(t_{n}^{2}\right)$, so the expected number of steps until stabilization is at most $t_{e}+t_{t}+t_{n}=O(1)+O\left(t_{n}^{2}\right)+t_{n}=O\left(t_{n}^{2}\right)$.

Now we use this bound on the expected number of steps to bound the expected real time until stabilization. Each decrement increases the "accuracy" species $A$. The slowest reaction in the system is a "jump" of the form $L_{i}+C_{4} \rightarrow L_{k}+C_{1}+A$. After $\ell$ instructions, the count of $A$ is at most $\ell$ (assuming in the worst case that every executed instruction is a decrement). Lemma A.4 (or Lemma 8 in the journal version) of [16] shows that with a clock module having $s$ stages, the expected time for the $\ell^{\prime}$ th decrement to occur is at most $O\left(\ell^{s-1}\right)$, which is $O\left(\ell^{3}\right)$ since $s=4$ in our case. ${ }^{10}$ Therefore, all $t_{n}^{2}$ steps take total expected time $O\left(\sum_{\ell=1}^{O\left(t_{n}^{2}\right)} \ell^{3}\right)=O\left(t_{n}^{8}\right)$.

The proof of Theorem 4.1 assumes a constant volume. The construction is set up to balance the forward and reverse rates of the clock module by using the constant-rate unimolecular reaction $C_{i} \rightarrow C_{i+1}$ to advance the random walk forward and the "growing with \# $A$ "-rate bimolecular reaction $C_{i+1}+A \rightarrow C_{i}+A$ to take it backward, giving a reverse bias to the random walk. If we instead obeyed the finite density constraint — discussed in Section 2 - by assuming the volume grows to remain proportional to the current molecular count, then the count of $A$ eventually dominates the volume requirement since it is the only species to grow unboundedly. ( $T$ can grow

\footnotetext{
${ }^{10}$ They actually show it is $O\left(\ell^{s-1} v / k\right)$, where $v$ is the volume ( 1 in our case), $k$ is the rate constant on all reactions (also 1 in our case).
} 
unboundedly but with probability 1 it eventually stops.) In this case, the rate of the reaction $C_{i+1}+A \rightarrow C_{i}+A$ would be $\Theta(1)$. To create the reverse bias required in the random walk for the proof of Theorem 4.1 to work, it suffices to alter the forward reactions to have a catalyst $F$ with count 1: $C_{i}+F \rightarrow C_{i+1}+F$. In this case, in volume $v=O(\# A)$, the rate of the forward reaction is $\frac{1}{v}$, which implies the same relative bias as in the current constant-volume construction (even though the absolute rates in both directions are now lower by a factor of $v$ ), leading to the same conclusion that with probability 1 , a finite number of errors occur. However, with volume $v=O(\# A)$ instead of $O(1)$, the expected time for the $\ell^{\prime}$ th decrement increases from $\ell^{3}$ to $\ell^{4}$ (since the expected time given by Lemma A.4 of [16] is $O\left(\# A^{3} \cdot v\right)$ ). Thus the expected time until stabilization increases from $O\left(\sum_{\ell=1}^{O\left(t_{n}^{2}\right)} \ell^{3}\right)=O\left(t_{n}^{8}\right)$ to $O\left(\sum_{\ell=1}^{O\left(t_{n}^{2}\right)} \ell^{4}\right)=O\left(t_{n}^{10}\right)$.

\section{$5 \quad \Delta_{2}^{0}$ predicates}

In this section we extend the technique of Section 3 to show that every $\Delta_{2}^{0}$ predicate is decidable with probability 1 by a CRD (Theorem 5.1). We also show the converse result (Theorem 5.2) that every predicate decidable with probability 1 by a CRD is in $\Delta_{2}^{0}$. Theorems 5.1 and 5.2 give our main result, that probability 1 decidability by CRDs is exactly characterized by the class $\Delta_{2}^{0}$.

Theorem 5.1. Every $\Delta_{2}^{0}$ predicate is decidable with probability 1 by a CRD.

Proof. For every $\Delta_{2}^{0}$ predicate $\phi: \mathbb{N} \rightarrow\{0,1\}$, there is a computable function $r: \mathbb{N} \times \mathbb{N} \rightarrow\{0,1\}$ such that, for all $n \in \mathbb{N}, \lim _{t \rightarrow \infty} r(n, t)=\phi(n)$. Therefore there is a register machine $M$ that computes $r$. As in Section 3, we first construct a register machine $S$ that simulates $M$ in a controlled fashion that ensures errors in simulation by a CRD will be handled gracefully.

We now argue that the definition of $\Delta_{2}^{0}$ does not change if we require the register machine $M$ computing $r(n, t)$ to halt in exactly $2 t$ steps. To see this, let $M$ be the register machine that computes $r$. Define the non-halting register machine $M^{\prime}$ that, on input $n$, does the following. For each $t^{\prime} \in \mathbb{N}, M^{\prime}(n)$ runs $M\left(n, t^{\prime}\right)$, updating its own output to be the output of $M\left(n, t^{\prime}\right)$ for the most recent value of $t^{\prime}$ for which $M\left(n, t^{\prime}\right)$ produced an output. Define the halting register machine $M^{\prime \prime}$ that, on input $n, t$ (each number stored in its own register $r_{1}$ and $r_{2}$, respectively), does the following. $M^{\prime \prime}(n, t)$ repeatedly decrements $r_{2}$ and then executes the next instruction of $M^{\prime}(n)$, updating its output to match the current output of $M^{\prime}$, and halting when $r_{2}$ reaches 0 . Clearly, $M^{\prime \prime}(n, t)$ halts in exactly $2 t$ steps (or $2 t+1$ if one counts the final halt as its own step). Also, as $t \rightarrow \infty$, the largest value of $t^{\prime}$ for which $M^{\prime}(n)$ simulates $M\left(n, t^{\prime}\right)$ also approaches $\infty$, which by the definition of $r$ implies that for sufficiently large $t, M^{\prime \prime}(n, t)$ is the correct output.

Similar to Section $3, S$ uses a "timer" to simulate $M(n, t)$ for at most $2 t$ steps. Unlike in Section $3, S$ decrements the timer after every step (not just the decrement steps) and the timer is incremented by 2 after each execution (in the previous construction, the timer is incremented by 1 and only if $M$ exceeds the time bound). Note that no matter what errors occur, no execution can go for longer than $2 t$ steps by the timer construction in Section 3. So $S$ will dovetail the computation as before, running $M(n, 1)$ for 2 steps, $M(n, 2)$ for 4 steps, etc., and in between each execution of $M(n, t)$, update its voting species with the most recent answer.

As in the construction of Section 3, so long as the d'th decrement has error probability at most $\frac{1}{d^{3}}$, then by the Borel-Cantelli lemma, with probability 1 a finite number of errors occur. Errors in the CRD simulating $S$ maintain the input without error and increment the timer value without error. Thus after the last error occurs, and after $t$ is sufficiently large that $r(n, t)=\phi(n)$, the CRD will stop updating its voter, and the CRD's output will be correct.

The next theorem shows that only $\Delta_{2}^{0}$ predicates are decidable with probability 1 by a CRD. 
Theorem 5.2. Let the $C R D \mathcal{D}$ decide predicate $\phi: \mathbb{N} \rightarrow\{0,1\}$ with probability 1. Then $\phi \in \Delta_{2}^{0}$.

Proof. It suffices to show that there is a computable function $r: \mathbb{N} \times \mathbb{N} \rightarrow\{0,1\}$ such that, for all $n \in \mathbb{N}, \lim _{t \rightarrow \infty} r(n, t)=\phi(n)$. The function $r$ is defined for $n, t \in \mathbb{N}$ as $r(n, t)=1$ if the probability that $\mathcal{D}$ is outputting "yes" after exactly $t$ reactions have occurred is at least the probability that $\mathcal{D}$ is outputting "no," when started in an initial state encoding $n$, and $r(n, t)=0$ otherwise.

To see that $r$ is computable, consider the Turing machine $M$ that, on input $(n, t)$, searches the set of states reachable from the initial state $\mathbf{i}$ of $\mathcal{D}$ (with $n$ copies of its input species) by executing exactly $t$ reactions. Call $t$ the depth of the state; we consider identical states that are encountered at different depths of the search differently. If $\mathbf{c}$ is reachable from $\mathbf{i}$ in exactly $t$ reactions, then write $\mathbf{c} \in \mathrm{REACH}^{t}(\mathbf{i})$.

Each (state,depth) pair $(\mathbf{c}, t)$ is assigned a measure $\mu: \mathbb{N}^{\Lambda} \times \mathbb{N} \rightarrow[0,1]$ recursively as follows. ${ }^{11}$ The initial state $(\mathbf{i}, 0)$ has $\mu(\mathbf{i}, 0)=1$, and all other states $\mathbf{c}$ have $\mu(\mathbf{c}, 0)=1$. If state $\mathbf{c}$ is reachable by exactly 1 reaction from exactly $\ell$ states $\mathbf{c}_{1}, \ldots, \mathbf{c}_{\ell}$, each of which are reachable from $\mathbf{i}$ by exactly $t-1$ reactions, with $\mathbf{c}_{j}$ followed by a single reaction that occurs as the next reaction in state $\mathbf{c}_{j}$ with probability $p_{j}$ to reach state $\mathbf{c}$, then

$$
\mu(\mathbf{c}, t)=\sum_{j=1}^{\ell} p_{j} \cdot \mu\left(\mathbf{c}_{j}, t-1\right) .
$$

That is, $\mu(\mathbf{c}, t)$ is the probability that after exactly $t$ reactions have occurred from the initial state $\mathbf{i}, \mathcal{D}$ is in state $\mathbf{c}$. Each value $p_{j}$ is a rational number computable from the counts of species in state $\mathbf{c}_{j}$, so $\mu(\mathbf{c}, t)$ is computable.

For $b \in\{0,1\}$, let

$$
\mu_{b}(t)=\sum_{\substack{\mathbf{c} \in \mathrm{REACH}^{t}(\mathbf{i}) \\ \Phi(\mathbf{c})=b}} \mu(\mathbf{c}, t) .
$$

That is, $\mu_{b}(t)$ is the probability that, after exactly $t$ reactions, the output of $\mathcal{D}$ is $b$. If $\mathcal{D}$ decides $\phi$ with probability 1 , then on input $n$ with $\phi(n)=b$, we have $\lim _{t \rightarrow \infty} \mu_{b}(t)=1$ and $\lim _{t \rightarrow \infty} \mu_{1-b}(t)=0$.

$M$ computes $\mu_{0}(t)$ and $\mu_{1}(t)$ and outputs 1 if $\mu_{1}(t) \geq \mu_{0}(t)$ and outputs 0 otherwise. Due to the limit stated above, for all sufficiently large $t, M(n, t)$ outputs $\phi(n)$, whence $\phi \in \Delta_{2}^{0}$.

Acknowledgements. We thank Shinnosuke Seki, Chris Thachuk, and Luca Cardelli for many useful and insightful discussions.

\footnotetext{
${ }^{11}$ Technically, we are defining, for each $t \in \mathbb{N}$, a measure on the set of all states, giving the state's probability of being reached in exactly $t$ steps, so for each $t \in \mathbb{N}, \mu(\cdot, t): \mathbb{N}^{\Lambda} \rightarrow[0,1]$ is a probability measure on $\mathbb{N}^{\Lambda}$. Since the evolution of the system is Markovian, once we know the probability $\mu(\mathbf{c}, t)$ of ending up in state c after precisely $t$ steps, it does not matter the particular sequence of $t-1$ states preceding $\mathbf{c}$ that got the system there, in order to determine probability of the various states that could follow c.
} 


\section{References}

[1] Dana Angluin, James Aspnes, and David Eisenstat. Stably computable predicates are semilinear. In PODC 2006: Proceedings of the twenty-fifth annual ACM symposium on Principles of distributed computing, pages 292-299, New York, NY, USA, 2006. ACM Press.

[2] Dana Angluin, James Aspnes, and David Eisenstat. Stably computable predicates are semilinear. In PODC 2006: Proceedings of the twenty-fifth annual ACM symposium on Principles of distributed computing, pages 292-299, New York, NY, USA, 2006. ACM Press.

[3] James Aspnes and Eric Ruppert. An introduction to population protocols. Bulletin of the European Association for Theoretical Computer Science, 93:98-117, 2007.

[4] Robert Brijder. Output stability and semilinear sets in chemical reaction networks and deciders. In DNA 2014: Proceedings of The 20th International Meeting on DNA Computing and Molecular Programming, Lecture Notes in Computer Science. Springer, 2014.

[5] Luca Cardelli. Strand algebras for DNA computing. Natural Computing, 10(1):407-428, 2011.

[6] Ho-Lin Chen, David Doty, and David Soloveichik. Deterministic function computation with chemical reaction networks. Natural Computing, 2013. to appear. Preliminary version appeared in DNA 2012.

[7] Yuan-Jyue Chen, Neil Dalchau, Niranjan Srinivas, Andrew Phillips, Luca Cardelli, David Soloveichik, and Georg Seelig. Programmable chemical controllers made from DNA. Nature Nanotechnology, 8(10):755-762, 2013.

[8] Matthew Cook, David Soloveichik, Erik Winfree, and Jehoshua Bruck. Programmability of chemical reaction networks. In Anne Condon, David Harel, Joost N. Kok, Arto Salomaa, and Erik Winfree, editors, Algorithmic Bioprocesses, pages 543-584. Springer Berlin Heidelberg, 2009 .

[9] William Feller. An Introduction to Probability Theory and Its Applications, volume 1. Wiley, January 1968.

[10] Daniel T. Gillespie. Exact stochastic simulation of coupled chemical reactions. Journal of Physical Chemistry, 81(25):2340-2361, 1977.

[11] Richard M Karp and Raymond E Miller. Parallel program schemata. Journal of Computer and system Sciences, 3(2):147-195, 1969.

[12] Carl A Petri. Communication with automata. Technical report, DTIC Document, 1966.

[13] Hartley Rogers, Jr. Theory of Recursive Functions and Effective Computability. McGrawHill Series in Higher Mathematics. McGraw-Hill Book Company, New York-St. Louis-San Francisco-Toronto-London-Sydney-Hamburg, 1967.

[14] Joseph R. Shoenfield. On degrees of unsolvability. Annals of Mathematics, 69(3):644-653, 1959.

[15] Robert Irving Soare. Interactive computing and relativized computability. Computability: Turing, Gödel, Church, and beyond (eds. BJ Copeland, CJ Posy, and O. Shagrir), MIT Press, Cambridge, MA, pages 203-260, 2013. 
[16] David Soloveichik, Matthew Cook, Erik Winfree, and Jehoshua Bruck. Computation with finite stochastic chemical reaction networks. Natural Computing, 7(4):615-633, 2008.

[17] David Soloveichik, Georg Seelig, and Erik Winfree. DNA as a universal substrate for chemical kinetics. Proceedings of the National Academy of Sciences, 107(12):5393, 2010. Preliminary version appeared in DNA 2008.

[18] Vito Volterra. Variazioni e fluttuazioni del numero dindividui in specie animali conviventi. Mem. Acad. Lincei Roma, 2:31-113, 1926.

[19] Gianluigi Zavattaro and Luca Cardelli. Termination problems in chemical kinetics. CONCUR 2008-Concurrency Theory, pages 477-491, 2008. 